Convergences francophones 6.2 (2020): 95-104

http://mrujs.mtroyal.ca/index.php/cf/index

\title{
Langue et identité : la construction identitaire des victimes de guerre
}

\author{
Francis Apasu et Amal Madibbo \\ Université de Calgary
}

La langue fait partie intégrante de la construction de l'identité individuelle et collective, car tout être humain a besoin de communiquer avec le monde qui l'entoure pour exprimer ses besoins fondamentaux. Dans l'exercice de cette capacité langagière, il cherche à s'identifier, et à être compris, apprécié et à comprendre autrui. Toujours est-il que cet échange reflète la compétence communicative des personnes impliquées dans la conversation et la performance langagière. Cette compétence n'est pas le fait du hasard, car elle est délicatement construite avec les valeurs éthiques et esthétiques de la société où est né, a grandi et évolue le locuteur. C'est un amalgame d'expériences et de vécus quotidiens qui constitue cette compétence langagière. Selon Arthur «le signifiant entretient un rapport intime avec la position éthique de celui qui le parle ; il représente le sujet » (127). Partant de cette réflexion, il est évident que la langue n'est pas uniquement destinée à exprimer le contenu informationnel d'un signe linguistique, elle révèle également l'identité des interlocuteurs, ce qu'ils ont vécu et les séquelles de leurs expériences. Cette révélation profonde du for intérieur du locuteur par ses énoncés expose le rôle identitaire de la langue et démontre la relation intrinsèque entre le discours et l'identité. Si nous admettons avec Charaudeau que «pour traiter de l'identité culturelle à travers les faits de langage, il faut se référer à ce qu'est la compétence langagière » (3), nous nous posons les deux questions suivantes : 1) Comment l'identité se construit-elle à travers la langue ? et 2) À quoi la compétence et la performance langagières se réfèrent-elles dans ce rapport biunivoque entre la langue et l'identité?

Pour répondre à ces questions, nous allons, dans un premier temps, mettre en relief le rapport entre la langue et l'identité en soulevant les éléments constitutifs de l'identité et les rapports qu'ils entretiennent entre eux, surtout en nous servant des travaux de Charaudeau, Ferret et Leclerc. Puis, nous allons explorer les dimensions de la notion de compétence communicative et de la performance langagière dans les perspectives chomskyennes. La troisième partie nous amènera à dégager le rapport entre les expériences vécues, la forme du contenu conversationnel et le rapport entre la langue et l'identité chez les victimes de guerre, chez des ressortissants du Soudan du Sud qui ont immigré au Canada. ${ }^{1}$

\footnotetext{
${ }^{1}$ Les entretiens ont été effectués dans le cadre du projet « Immigrant Transnationalism and its Impacts on the Sense of Belonging to Canada: The Case of Africans in Calgary » qui a été subventionné par le Conseil de recherches en sciences humaines du Canada (2016-2019). L'équipe de recherche - Francis Apasu, Animwaa Obeng-Akrofi, Mabel Teye-Kau, Yusuf Shoelye et Dr. Amal Madibbo - a recueilli les données de l'étude selon des méthodes de recherches qualitatives d'entretiens semi-directifs et d'analyses documentaires. Les résultats présentés ici concernent quatre des trente-deux ressortissants de cinq pays africains qui ont participé à cette étude. Deux tiers des entretiens ont été réalisés en français. L'entretien d'Aluel cité ici a été entamé en français.
} 


\section{Langue et identité}

La langue, ce moyen par lequel l'être humain arrive à interagir avec le monde qui l'entoure, est une représentation symbolique et indissociable de l'identité. Au fait, les constituants de l'identité transcendent le cadre individuel et englobent l'ensemble de la société. Comme l'a bien souligné Ferret (1), « la notion d'identité s'est, en même temps, communautarisée en glissant nettement d'une dimension individuelle à une dimension collective ». Cette dimension sociale du langage humain relève du fait que tous les liens interpersonnels dans la société sont délicatement construits à l'échelle d'un consensus collectif. Chaque groupe d'individus a besoin de cette unité pour évoluer dans le temps et dans l'espace (Charaudeau 341-348). La langue est avant tout un consensus ou un contrat entre les membres d'une communauté (Leclerc 28-34). La société construit le signifiant et le signifié de chaque entité lexicale suivant ses besoins et les relations interpersonnelles de ses membres. Cela étant, il est facile de déduire le comportement d'une communauté linguistique à partir des expressions d'un seul individu de cette communauté et vice versa. Sur le plan culturel, les langues contiennent en elles la culture de ceux qui la parlent et forment, par là, leur identité culturelle. Qui parle d'une langue parle d'une civilisation, d'une histoire et d'une société donnée. Cette identité culturelle est la somme du savoir-faire, du savoir-être et de la croyance d'un peuple donné. C'est dans ces perspectives que Belgacem pense que «l'identité culturelle est un processus [qui se fait] en fonction, d'une part, d'adhésions et d'identifications aux manières de faire, d'être, de penser d'une communauté » (52) et, d'autre part, des divergences, «d'exclusions relatives aux manières de faire, d'être, de penser des communautés voisines » (52). La langue peut être alors perçue comme un miroir qui reflète l'identité de l'ensemble d'une communauté donnée.

Ce faisant, la langue marque et décrit le savoir-faire et le savoir-être de chaque individu tout en singularisant la valeur de celles des autres dans la société où il se trouve. Encore, est-il vrai que chaque individu possède sa manière de parler au sein d'une même communauté linguistique. Évidemment, toute parole individuelle a ses caractéristiques qui lui sont propres, quelle que soit la similitude de l'aperture d'articulation des sons des membres de la communauté linguistique où le locuteur réside. Les éléments distinctifs et individuels de la parole ne sont pas l'effet du hasard : elles se construisent minutieusement dès la petite enfance (Boysson-Bardies 1478). Cependant, ce fonctionnement individuel est ancré dans un environnement social où les traits phonologiques de la langue se développent et se perfectionnent par le biais d'une interaction interpersonnelle.

Étant donné que toute la connaissance humaine est ancrée dans le langage, toute construction du savoir, du savoir-faire et du savoir-être ne serait possible que par le biais d'une langue donnée. Même le nom, cette étiquette qu'on accroche aux gens, participe à la construction de l'identité individuelle. Le nom porte toujours en lui les éléments d'une civilisation relatifs à une ou plusieurs communautés linguistiques puisqu'il est nécessairement composé d'un signifiant et d'un signifié qui relève de la société. En dehors de cette relation sociale, le nom demeure la 
propriété exclusive de la personne qui le porte. Ainsi, le nom peut être défini comme un signe qui singularise les êtres humains dans la société et constitue leur première marque identitaire. Le nom a une relation intrinsèque avec la partie psychique de la personne qui le porte, car, lorsque le nom d'une personne est prononcé, il y a déjà quelque chose de psychique qui est déclenché en elle. La simple prononciation du nom va irrémédiablement traduire un changement soudain dans l'expression physionomique de la personne qui le porte. L'identité patronyme a alors un effet d'émotion explicite sur les êtres humains. Si nous convenons avec Costalat-Founeau que l'identité « renvoie à des notions psychologiques comme image ou représentation de soi, perception de soi, sentiment de soi, estime de soi » (6), et que le nom influe sur nos sentiments, alors nous pouvons admettre que le nom fait partie de la construction de l'identité. Nous étendons ces perspectives au nom de manière générale telle l'appellation des régions, pays ou expériences vécues, comme la guerre ou l'exil. Ces noms ont un impact sur l'identité parce qu'ils évoquent des émotions et des souvenirs liés à ce qu'on est ou a été.

D'un point de vue global, la langue fait partie intégrante des éléments constitutifs de l'identité humaine. En effet, la construction identitaire commence par la langue et se termine par elle ; elle révèle explicitement toute la connaissance et la capacité intellectuelle du locuteur vis-à-vis du monde qui l'entoure. Ainsi, la langue donne une information panoramique sur la culture, la croyance, l'éducation et tout ce qui constitue l'identité de celui qui la parle à ses interlocuteurs. Cependant, la langue n'arrive pas parfois à dévoiler toutes les compétences linguistiques acquises par le locuteur. Le contexte de communication et les valeurs socioculturelles font perdre, ou ajouter, certaines rigueurs au message source. À cela viennent s'ajouter les expériences et les vécus quotidiens du locuteur. Dans ce cas, le message exprimé n'équivaut pas au message source, le message source étant certainement plus riche et intelligible que le message exprimé. C'est dans ce sens que Chomsky (32-58) établit la différence entre deux éléments langagiers qui déterminent l'identité, ce que nous connaissons (la compétence) et la manière dont nous le communiquons aux gens (la performance).

\section{La compétence, la performance et l'identité}

Chomsky fait émerger ces notions de compétence et performance pour établir la différence entre les compétences linguistiques acquises et le message exprimé (3258). Chomsky définit le concept de compétence, d'une part, comme une possibilité illimitée de construire ou de comprendre un nombre infini de phrases et, d'autre part, comme la possibilité de porter un jugement de grammaticalité sur une phrase, de comparer des degrés de grammaticalité ou de déceler des ambiguïtés. Il s'agit d'une structure de savoir linguistique qu'un individu maîtrise et connaît, savoir qui le rend capable de parler, de comprendre et de juger de l'acceptabilité d'une phrase. En clarifiant cette pensée de Chomsky davantage, Vivier fait valoir que :

La compétence se réduit à une liste d'unités définies par leurs caractéristiques formelles (article, nom, verbe, groupe nominal...) et une suite de règles pour décrire les phrases à l'aide de ces unités. Le but est de 
parvenir par réécriture à un minimum de règles explicatives suffisantes pour générer un ensemble de structures sous-jacentes. Il suffira d'ajouter l'insertion lexicale et des transformations obligatoires pour que le canevas obtenu se transforme en phrase réelle véhiculant une signification complète (11)

La compétence est l'acquisition des structures qui constituent le fonctionnement d'une langue donnée, alors que la performance est la mise en œuvre de cette acquisition, dans une situation de communication. De fait, la performance est l'acte qui illustre la compétence. Cette tendance générativiste révèle que c'est au cours de la performance que chaque personne arrive à extérioriser son identité individuelle ou collective. L'analyse des énoncés des répondants nous permet d'explorer ces hypothèses.

\section{Les conséquences des expériences sur la langue et l'identité}

Notre population de référence comprend quatre personnes originaires du Soudan du Sud. Elles ont vécu la deuxième guerre civile du Soudan (1983-2005) d'une manière directe ou indirecte et vivent toutes à Calgary au moment de l'entretien. Nous allons dégager leurs trajectoires identitaires ainsi que leur compétence et performance langagières afin d'établir le rapport entre leur discours et leur identité.

Tableau 1: Le profil identitaire des répondants ${ }^{2}$

\begin{tabular}{|c|c|c|c|c|c|}
\hline Nom & $\begin{array}{c}\text { Pays } \\
\text { d'origine }\end{array}$ & $\begin{array}{c}\text { Résidence } \\
\text { pendant la } \\
\text { guerre }\end{array}$ & $\begin{array}{c}\text { Age avant } \\
\text { la guerre }\end{array}$ & $\begin{array}{c}\text { Première } \\
\text { langue } \\
\text { (L1) }\end{array}$ & $\begin{array}{c}\text { Activités avant } \\
\text { la guerre }\end{array}$ \\
\hline John & $\begin{array}{c}\text { Soudan du } \\
\text { sud }\end{array}$ & $\begin{array}{c}\text { Soudan du } \\
\text { Sud }\end{array}$ & 10 ans & Dinka & $\begin{array}{c}\text { Agricoles \& } \\
\text { pastorale }\end{array}$ \\
\hline Jennifer & $\begin{array}{c}\text { Soudan du } \\
\text { Sud }\end{array}$ & $\begin{array}{c}\text { Soudan du } \\
\text { Sud }\end{array}$ & $\begin{array}{c}\text { Moins (-) } \\
\text { de } 10 \text { ans } \\
(10)\end{array}$ & Dinka & Élève \\
\hline Chol & $\begin{array}{c}\text { Soudan du } \\
\text { Sud }\end{array}$ & Egypte & - de 10 & Dinka & Ménagerie \\
\hline Aluel & $\begin{array}{c}\text { Soudan du } \\
\text { Sud }\end{array}$ & Egypte & 6 ans & $\begin{array}{c}\text { Dinka / } \\
\text { Arabe }\end{array}$ & Élève \\
\hline
\end{tabular}

Le tableau ci-dessus révèle que les quatre répondants de notre enquête sont tous nés au Soudan du Sud et y ont passé une partie de leur enfance. Ils ne viennent pas d'un même village, mais ils sont tous issus du même espace géographique, dans ce cas le Soudan du Sud. Quant à leur lieu de résidence avant l'éclatement de la guerre, deux de nos enquêtés, à savoir John et Jennifer, vivaient au Soudan du Sud avant le début de la deuxième guerre civile du Soudan, le 5 juin 1983, tandis que les autres, Chol et Aluel, ont immigré en Égypte pour rejoindre leurs familles qui avaient fui à cause de la première guerre civile du Soudan (1957-1972). Ils étaient

\footnotetext{
${ }^{2}$ Nous avons employé des pseudonymes pour respecter la confidentialité des répondants.
} 
tous enfants quand la guerre a éclaté et ils possèdent tous une même langue maternelle, le dinka, bien que certains aient développé d'autres compétences dans le milieu où ils vivaient, comme c'est le cas d'Aluel qui a précisé qu'elle parlait couramment l'arabe et qu'elle a appris le français en Égypte.

Tableau 2 : La compétence langagière des répondants

\begin{tabular}{|c|c|c|c|c|c|}
\hline \multirow[t]{2}{*}{ Nom } & \multicolumn{4}{|c|}{ Éducation au Canada } & \multirow[b]{2}{*}{$\begin{array}{c}\text { Nombre } \\
\text { d'années au } \\
\text { Canada }\end{array}$} \\
\hline & $\begin{array}{l}\text { Anglais } \\
\text { Langue } \\
\text { Seconde } \\
\text { (ALS) }\end{array}$ & Lycée & $\begin{array}{c}\text { Études } \\
\text { universitaires }\end{array}$ & $\begin{array}{l}\text { Autres } \\
\text { qualifications } \\
\& \text { Leadership }\end{array}$ & \\
\hline John & $\sqrt{ }$ & - & $\begin{array}{l}\text { Maîtrise en } \\
\text { sciences } \\
\text { économiques }\end{array}$ & - & 16 ans \\
\hline Jennifer & $\sqrt{ }$ & - & $\begin{array}{l}\text { License en } \\
\text { sciences } \\
\text { économiques }\end{array}$ & $\begin{array}{l}\text { Président des } \\
\text { associations } \\
\text { des étudiants }\end{array}$ & 12 ans \\
\hline Chol & $\sqrt{ }$ & & $\begin{array}{l}\text { Licence en } \\
\text { sciences } \\
\text { sociales }\end{array}$ & - & 13 ans \\
\hline Aluel & & $\sqrt{ }$ & Étudiante & $\begin{array}{l}\text { Travail } \\
\text { Saisonnier }\end{array}$ & $\begin{array}{c}\text { Plus de } 11 \\
\text { ans }\end{array}$ \\
\hline
\end{tabular}

Quant à la compétence langagière des répondants dans la deuxième langue (L2), dans ce cas l'anglais, ils ont tous suivi des cours d'anglais langue seconde dans différentes écoles de langues au Canada, avant de commencer leurs études supérieures. Ils auraient au moins atteint le niveau B2 du Cadre européen commun de référence pour les langues, ce qui implique qu'ils étaient capable d' « utiliser la langue avec aisance, correction et efficacité dans une gamme étendue de sujets d'ordre général, éducationnel, professionnel » (Conseil de l'Europe 61). Le programme universitaire auquel ils sont inscrits requiert quatre années au moins pour terminer la formation. Les cours sont irrémédiablement donnés en anglais, ce qui permet aux participants d'être en contact permanent avec l'anglais académique, durant les quatre années d'études. La dernière colonne, qui parle de la durée de résidence de chacun des répondants au Canada, révèle le contact informel ou naturel avec la langue anglaise. L'anglais reste la langue dominante dans les conversations quotidiennes dans la province de l'Alberta où les répondants résident. Conséquemment, les répondants ont une expérience variant de 12 à 16 ans dans un milieu linguistique anglais. Nous pouvons donc admettre que les répondants ont un atout indiscutable pour pratiquer et développer ce qu'ils apprennent dans les cours de langues dans la société (Canale \& Merril 35). Théoriquement, malgré de petites nuances, tous les répondants sont censés obtenir presque la même compétence langagière en anglais. Cependant, on pourrait se demander s'ils peuvent réagir de la même manière dans une situation de communication réelle. 
Convergences francophones 6.2 (2020): 95-104

http://mrujs.mtroyal.ca/index.php/cf/index

Tableau 3 : La performance langagière des répondants

\begin{tabular}{|c|c|c|c|}
\hline Nom & Événements & Phrases normales & $\begin{array}{c}\text { Phrases } \\
\text { anormales }\end{array}$ \\
\hline \multirow{2}{*}{ John* } & Heureux \& neutres & ++ & - \\
\cline { 2 - 4 } & Malheureux & - & +++ \\
\hline \multirow{2}{*}{ Jennifer* } & Heureux \& neutres & +++ & - \\
\cline { 2 - 4 } & Malheureux & - & +++ \\
\hline \multirow{2}{*}{ Chol** } & Heureux \& neutres & +++ & + \\
\cline { 2 - 4 } & Malheureux & + & - \\
\hline \multirow{2}{*}{ Aluel** } & Heureux \& neutres & +++ & +++ \\
\cline { 2 - 4 } & Malheureux & + & + \\
\hline
\end{tabular}

$*=$ Les participants ayant vécu la guerre civile du Soudan du Sud. $* *=$ Les participants n'ayant pas vécu la guerre. - = absence du type de phrase. $+=$ Présence de type de phrase. $++=$ Présence fréquente de type de phrase. $+++=$ Très forte présence de type de phrase.

L'échantillon des énoncés des répondants nous permet de classer leurs énoncés en deux groupes : les phrases dites «normales» et les phrases dites « anormales ». Selon notre classement, les phrases normales sont celles qui suivent la structure morphosyntaxique de l'anglais, alors que les phrases anormales sont des phrases qui sont jalonnées de répétitions d'hésitations, de structures agrammaticales, etc. Nous verrons que ces énoncés cernent le lien entre la compétence et la performance langagières chez les répondants et que l'expérience vécue, dans ce cas la guerre, se répercute dans leur construction identitaire.

\section{Les victimes directement impliquées dans la guerre}

À la question « What were you doing when you were young? », ${ }^{3}$ John répond en ses termes: " Before the war broke out, I was a cattle herder. I used to take care of my father's cattle ». ${ }^{4}$ Non seulement, la structure de cette phrase est « normale », elle répond aux critères des règles de morphosyntaxe anglaise, mais elle donne aussi une compréhension claire et logique du message, une certaine cohérence, une bonne concordance des temps verbaux dans la phrase. Cependant, les phrases changent de formes lorsque John répond à la question portant sur la manière dont il a fui devant les soldats armés : «No, like you know, and like a lot of I that... think in that book he mentioned a lot of,... a lot of people were drowned in the river called Akobo and we tried like... »..$^{5}$ Cette phrase est «anormale », car elle laisse entrevoir, d'une part, une mauvaise disposition des unités linguistiques avec une séquence d'hésitations et de répétitions rendant ainsi la phrase difficile à

\footnotetext{
3 «Que faisais-tu quand tu étais jeune?»

${ }^{4}$ « Avant le début de la guerre, j'étais berger. Je prenais soin du bétail de mon père. »

5 « Non, comme tu le sais, et comme beaucoup de personnes comme moi... pensent dans ce livre dont il a mentionné beaucoup de choses, ... beaucoup de gens se sont noyés dans la rivière Akobo et nous avons essayé comme...»
} 
comprendre et, d'autre part, une réponse qui ne correspond pas à la question posée. Certes, la première phrase de John diffère de la dernière par le style, la structure et la cohérence.

Signalons que le style de Jennifer n'a pas fait exception dans cette gamme des victimes qui étaient directement impliquées dans la guerre. Les répétitions, les hésitations et les phrases agrammaticales se sont reproduites tout le long de ses réponses concernant la guerre ou les noms liés à la guerre. À la question «Where were you staying? In a camp? $»^{6}$, Jennifer répond:

In a camp, yes in a camp... Itang is a camp, a refugee camp. And there was another place called Gambela [Okugo Refugee Camp in Gambela]. They were there, many of the Lost Boys [of Sudan] were there. And then, I stayed with my mum. And there was a little school. When is time ... like the summer time. ${ }^{7}$

Comme chez John, Jennifer a mentionné le mot « camp » d'une manière répétitive au début de l'énoncé. La répétition du mot « camp » quatre fois ne révèle rien d'autre que les souvenirs désagréables de la guerre et montre l'image horrible que Jennifer associe à ce nom. Pour elle, les camps d'Itang et de Gambela sont des entités linguistiques qui traînent derrière elles les atrocités de la guerre. De fait, il se dégage de cette analyse que les répondants ayant eu un contact direct avec la guerre changent de style langagier chaque fois qu'on leur pose des questions relatives à l'atrocité de la guerre. Cette production fréquente de phrases « anormales» montre que la guerre a affecté la performance langagière des victimes directement impliquées dans ce conflit d'une façon qui donne lieu aux hésitations et expressions agrammaticales, donc à une performance qui n'est pas cohérente.

\section{Les victimes indirectement impliquées de la guerre}

Les répondants qui n'ont pas directement connu la guerre parce qu'ils vivaient au Caire avant ou pendant le déclenchement de celle-ci n'ont fourni que des énoncés intelligibles, cohérents et grammaticaux tout au long de leur conversation. Le style est presque stable dans toutes les circonstances, que ce soit un événement heureux ou malheureux, les répondants de ce groupe réagissent presque de la même manière. À titre d'exemple, pour la question « Did you go to school in Egypt before that time? $»^{8}$, Chol répond:

Yeah, I would not call it school. I will call it time management because during that time it wasn't an accredited school. We stopped in certain areas

\footnotetext{
${ }^{6}$ « Où étiez-vous? Dans un camp?»

${ }^{7}$ « Dans un camp, oui dans un camp ... Itang est un camp, un camp de réfugiés. Et il y avait un autre endroit appelé Gambela [le Camp de réfugiés d'Okugo à Gambel]. Ils étaient là, beaucoup de garçons perdus [du Soudan] étaient là. Et puis, je suis restée avec ma mère. Et il y avait une petite école. Quand est-ce que ... l'heure ressemble à l'heure d'été... »

8 «Es-tu allé à l'école en Égypte avant ce temps?»
} 
such as junior high, but then you cannot go more than that because the Egyptians, that time, didn't allow refugees to go to their schools. ${ }^{9}$

Dans cette réponse, on observe un certain niveau de grammaticalité et d'intelligibilité dans l'information donnée. Il en va de même dans les énoncés évoquant les événements malheureux. Par exemple, lorsque l'on pose la question " Is your mum here in Calgary? " ${ }^{10}$ à Chol, il répond en ces termes: "Yes, could you imagine that my mother survived all the attacks of the rebels. It was a miracle! She walked for weeks to flee the conflict. It was during this terrible journey that three of her friends were killed... ${ }^{11}$. Durant toute sa conversation de près d'une heure, nous n'avons noté que des phrases bien structurées dignes du niveau B2.

Aluel, de son côté, répondra à la question « De quoi te souviens-tu au sujet du Soudan? ? par « Je suis pas née là-bas. Je ne sais pas grand-chose à ce sujet, mais je connais des gens là-bas et je sais aussi qu'il y avait un conflit armé là-bas ». Le ton de cet énoncé est presque neutre et ne montre ni mélancolie ni stupéfaction. $\mathrm{Sa}$ réponse regroupe, dans un seul énoncé, sa naissance, ses relations interpersonnelles et la guerre. Ce qui implique que tous les mots de la phrase sont sur le même diapason et ne lui donne pas tellement de peine ou d'émotion vive. Même le mot "Soudan » ne déclenche rien en elle. Bien que la question puisse évoquer un certain niveau d'inquiétude, la structure morphosyntaxique de la phrase n'est pas "fautive". Le groupe de mots "Sudan » et " armed conflict » n'a pas suscité de vives émotions pouvant conduire à des phrases jalonnées de répétitions et d'hésitations.

Ceux qui vivaient au Caire n'ont entendu parler de la guerre qu'à la radio et à la télévision, par les on-dit et le témoignage des membres de leurs familles. Cette exposition indirecte ne semble pas avoir eu de conséquences linguistiques sur eux. En effet, on note l'absence de traumatisme et de mélancolie dans des discours « normaux », intelligibles et grammaticalement acceptables à des degrés différents tout au long de leurs conversations. A l'inverse, ceux qui ont directement vécu la guerre paniquent et changent de style quand il s'agit des événements relatifs aux atrocités de la guerre. Il y a là clairement un décalage entre la performance langagière des deux groupes. Cependant nous ne pouvons pas dire non plus que ces répondants n'ont pas la compétence langagière nécessaire pour s'exprimer en anglais, car ils ont fourni de bonnes phrases au moment de répondre à la question ayant rapport avec des événements heureux. Quoique tous les répondants aient la même origine et presque les mêmes apprentissages de l'anglais, ils ne réagissent pas de la même manière dans ces entretiens semi-directifs. Nous voyons dans cette

\footnotetext{
${ }^{9}$ « Ouais, je n'appellerais pas ça de l'école. Je parlerai de gestion du temps, car pendant ce temps, ce n'était pas une école accréditée. Nous nous sommes arrêtés à un certain niveau comme le collège, mais nous ne pouvions pas continuer, car les Égyptiens, à cette époque, n'autorisaient pas les réfugiés dans leurs écoles. »

10 « Est-ce que ta mère est ici à Calgary? »

${ }^{11}$ « Oui, pourriez-vous imaginer que ma mère a survécu à toutes les attaques des rebelles. C'était un miracle ! Elle a marché pendant des semaines pour fuir le conflit. C'est lors de ce terrible voyage que trois de ses amis ont été tués »
} 
analyse que les événements n'ont pas seulement laissé un traumatisme dans la vie des victimes, mais qu'ils ont aussi affecté leur performance langagière. Il est clair que l'environnement et tout ce qui s'y trouve influent sur la performance langagière des êtres humains, et participe ainsi à sa construction. Si la performance langagière, le véritable pivot de l'identité humaine, est modifiée par les expériences de la guerre, il est alors évident que ces expériences vécues entrent dans la construction de l'identité de ces victimes de la guerre.

\section{Conclusion}

Nous avons montré qu'il y a une relation de sous-ensembles qui se forme entre la langue, l'identité et la compétence et la performance langagières. Ainsi, pour la question « Comment l'identité se construit-elle à travers la langue ?», notre analyse a révélé que les expériences vécues influent sur la capacité langagière humaine et font changer inconsciemment le style langagier au moment où le locuteur se souvient d'un événement très désagréable. Dès lors, l'identité langagière du locuteur devient le résultat des expériences du passé et de celles du présent. Il devient évident que les éléments qui affectent le locuteur redéfinissent son comportement langagier. Comme la langue est l'élément central de l'identité, les facteurs qui affectent la langue influent également sur l'identité et sa construction. Dans ce sens, nous avons confirmé les témoignages des auteurs qui affirment qu'il y a un rapport étroit entre la langue et l'identité (Charaudeau 341-348). Quant à la

question « À quoi la compétence et la performance langagières se réfèrent-elles dans ce rapport biunivoque entre la langue et l'identité ?», nous avons mis en lumière que la compétence et la performance langagières exposent notre état d'âme au monde extérieur et deviennent, de cette façon, les éléments indiciels de notre identité. La compétence et la performance reflètent le niveau de la langue acquise, et la langue, à son tour, donne l'image identitaire du locuteur au monde qui l'entoure. Dans cette relation, la compétence est une acquisition cognitive des éléments linguistiques d'une langue donnée alors que la performance langagière se réfère à la production de l'énoncé, comme le souligne Chomsky (32-58). Dans notre étude, la performance langagière est une actualisation de la compétence langagière, ce qui corrobore également les idées de Chomsky (32-58). Notre analyse des témoignages des répondants en lien avec un contexte socioculturel spécifique comme celui de la guerre montre que les événements ne contribuent pas seulement à l'enrichissement de la connaissance linguistique du locuteur, mais qu'ils influent fréquemment sur la production du discours et, par la suite, sur la construction identitaire. 


\section{Bibliographie}

Arthur, Mary. « La fixion sectaire. » Recherches en psychanalyse, vol. 2, no. 12, 2011, pp. 124-129.

Belgacem, Dalila. «Identité et culture. Quelle construction identitaire pour l'enfant de migrant.» Les Cahiers Dynamiques, vol. 4, no. 57, 2012, pp. 51-56.

Boysson-Bardies, Bénédicte de. «Comment la parole vient à l'enfant. » Revue française de psychanalyse, vol. 71, no .5, 2007, pp. 1473-1480.

Canale, Michael \& Merril, Swain. « Theoretical bases of communicative approaches to second language teaching and testing. " Applied Linguistics, vol. 2, 1980, pp. 1-47.

Charaudeau, Patrick. « Langue, discours et identité culturelle. » Études de linguistique appliquée, vol. 3, no. 123-124, 2001, pp. 341-348.

Chomsky, Noam. Aspects de la théorie syntaxique. Paris : Editions du Seuil, 1971.

Conseil de l'Europe. Cadre européen commun de référence pour les langues : Apprendre, enseigner, évaluer. Strasbourg : Unité des politiques linguistiques, 2001.

Costalat-Founeau, Anne-Marie. " Identité, action et subjectivité. » Connexions, vol. 1 , no. 8, 2008, pp. 63-74.

Ferret, Carole. «L'identité, une question de définition. » Cahiers d'Asie centrale, 2011, pp. 19-20, mis en ligne le 01 janvier 2012, http://journals.openedition.org/asiecentrale/1516. Consulté le 30 juin 2016.

Leclerc, Jacques. Qu'est-ce- que la langue? Laval : Mondia, 1989.

Vivier, Jean. « De la compétence linguistique aux compétences langagières. » Problématique des cycles et recherche, vol. 5, 1992, pp. 9-25. 